\title{
Research on Application of Fuzzy Control Algorithm in Nonlinear System \\ Libo Yang
}

\author{
Guangdong University of Science \& Technology, Dongguan, Guangdong, 523083,china; \\ 654337610@qq.com
}

Keywords: Nonlinear System, Fuzzy Control Algorithm, Applied Analysis.

\begin{abstract}
As a kind of artificial intelligence technology, fuzzy control is widely used in the non-linear control system, and the application will be effective in industrial production, and then many large lags and nonlinear problems have been solved. This paper focuses on the research of the application of fuzzy control algorithm in nonlinear systems. With the advancement of science and technology, system automation is more and more strong, and to strengthen the automation system, it requires an in-depth study on the control system. Control system is an important direction of scientific research in China, and through the establishment of the basic structure and simulation, the depth of control analysis will be strengthened greatly. In system control, fuzzy control of nonlinear system is an important content. Through analysis and study of this control algorithm, it can provide effective fuzzy control of nonlinear systems. Based on the nonlinear system control algorithm, this system aims to analyze the advantages of the algorithm, thereby it is strengthening the level of application in practice.
\end{abstract}

\section{Introduction}

In many practical situations, nonlinear systems are difficult to be described by some known functions. The unavoidable uncertainties exist in dynamic systems, such as, system modeling error, influence of the working environment as well as the unknown physical phenomena. The neural networks or fuzzy systems have particularly effective way to deal with instability. Since the introduction of fuzzy set, people's judgment and thinking ability can be expressed by a simple digital expression, which makes the complicated system be solved by simple math principles, which also makes the solution of uncertain complicated problems possible. However, though there have been great breakthroughs, in the actual application of nonlinear systems, there are still many problems that need to be resolved. In the control study, the typical control that is based on the accurate model control of the controlled object is the control of the classical control and state space model control. In the actual research, besides the control that is based on object accurate control, there are more complex controls, the controlled object of this control is not clear, so the establishment of mathematical model is relative difficult. In order to achieve the effective use of this control, the establishment of mathematical model based on fuzzy control algorithm is the main method. Therefore, the active fuzzy control algorithm research of nonlinear system is of great significance.

This paper focuses on the application of fuzzy control technology, nonlinear control technology and controller structure design from fuzzy control algorithm and the work experiences, and the purpose is that the feasibility and high performance design method of control system can be realized, so the uncertain, diverse and complicated control system can become stable. Obviously, it is very important and significant for the development of nonlinear control theory.

\section{A Mathematical Description of Fuzzy Control}

Fuzzy control is an important category in the study of control, and this is not only a kind of real time control, but also it does not depend on the precise model of the controlled object, so it is a new computer control to break the tradition bound. This control has brought a new method to solve more complex computer problems. From the point of view of the characteristics, the demand upon model is relatively low, and the calculation in practical use is very simple, control performance is quite 
excellent. In nonlinear systems, the research of fuzzy control algorithm has used a nonlinear system formula to make the study simple.

$$
y(t+1)=0.5 y(t)+e^{|l-y(t)|} u(t)
$$

In this formula, u represents the input and y represents output, and the formula represents a large class of complex controlled objects that are difficult to be modeled in practice. According to this formula, when determining the reference trajectory, the control formula can be written.

\section{The principle of fuzzy control algorithm}

The principle of fuzzy control algorithm is the key content of the research, in the process of practical analysis, it mainly includes four aspects. The first one is to establish a fuzzy model of the non-linear system, then to achieve its standardization, making it into a parameter identification problem. For example, when considering a SISO nonlinear system, the system input space and output space are quantified according to the precision of the system, then the system characteristics will be transformed into a specific formula, and the formula reflects conditions for the system, and it also constitute a fuzzy model of the system.

The second one is online recursive correction on the model. In order to make the control calculation more precise, and the use of the new information is combined with linear identification theory results, and there is the online recursive test on the whole model, then making corresponding adjustments and changes, so that the accuracy of the model can be realized. The third one is to forecast error, and there is further correction of the output value. In the practical calculation, because the accuracy of the model is hard to control, there will be measuring errors, and the input value error will affect the output value, so there is a constant need for error prediction by using the minimum error as the basic algorithm of fuzzy control.

The fourth one is the image establishment of fuzzy control algorithm. Through the improvement of above steps, the specific algorithm of fuzzy control is deduced, and then constructing the calculation structure, and the fuzzy control could be further accurate with this structure. In short, the fuzzy control algorithm needs to deduce and modified step by step. Following the prescribed order, it will be more practical with that the algorithm. This is the principle diagram of fuzzy algorithm.

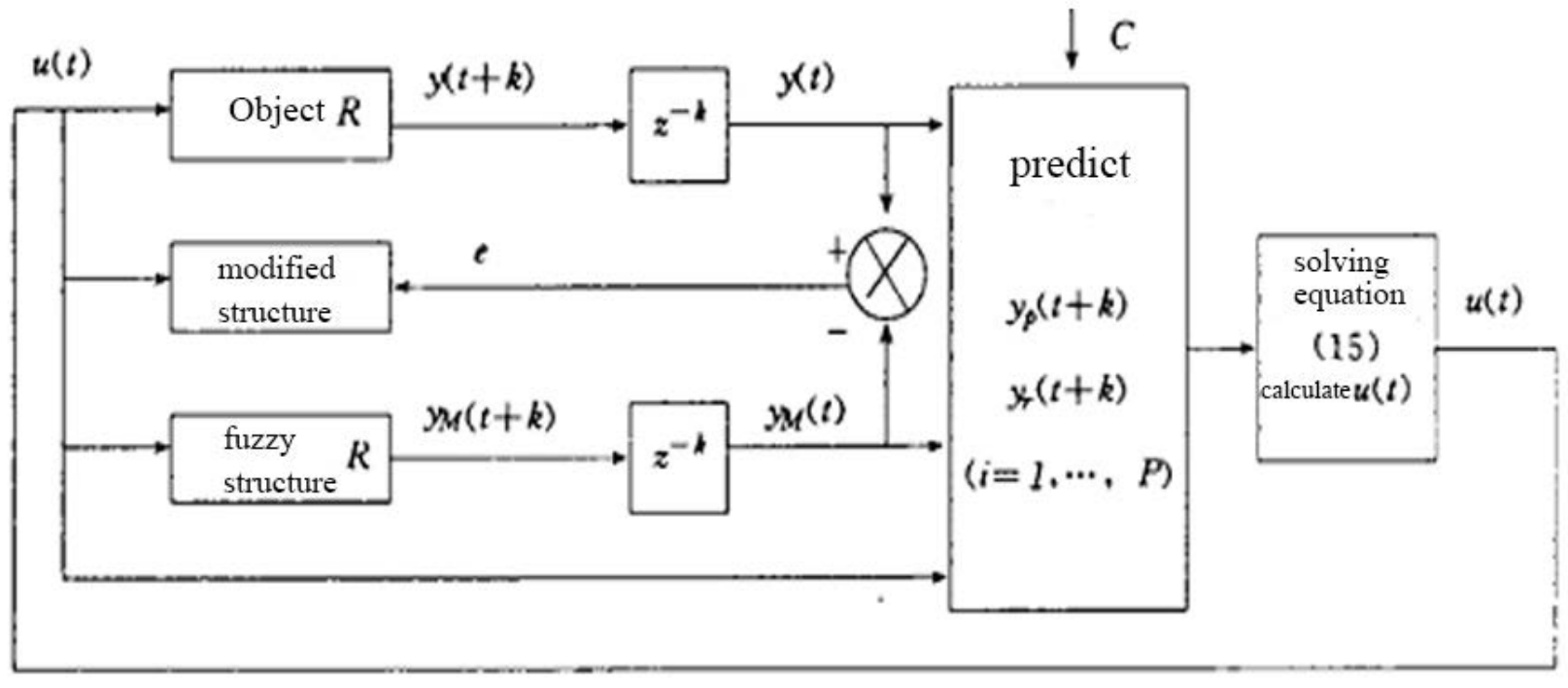

Fig.1 Schematic diagram of fuzzy algorithm

\section{Application of Fuzzy Control Algorithm in Nonlinear System}

According to the uncertainties of SISO matching conditions, a robust fuzzy control algorithm is proposed for nonlinear system. In this algorithm, the system state has been estimated with the design of observer. And there is not the requirement of testability state in hypothetical system (Figure 2, hypothetical result diagram). Based on this algorithm, it assumes the norm difference between 
optimal approximation nominal vector and parameter vector, and the related unknown bounds of approximation error. The robust design of fuzzy control algorithm must make all signals in the closed-loop system be in the bounded range, and the tracking error estimates will converge to a small zero area. From the proposed algorithm in application process to the simulation results of gearbox that is connected to the DC motor, all show the validity of the algorithm.

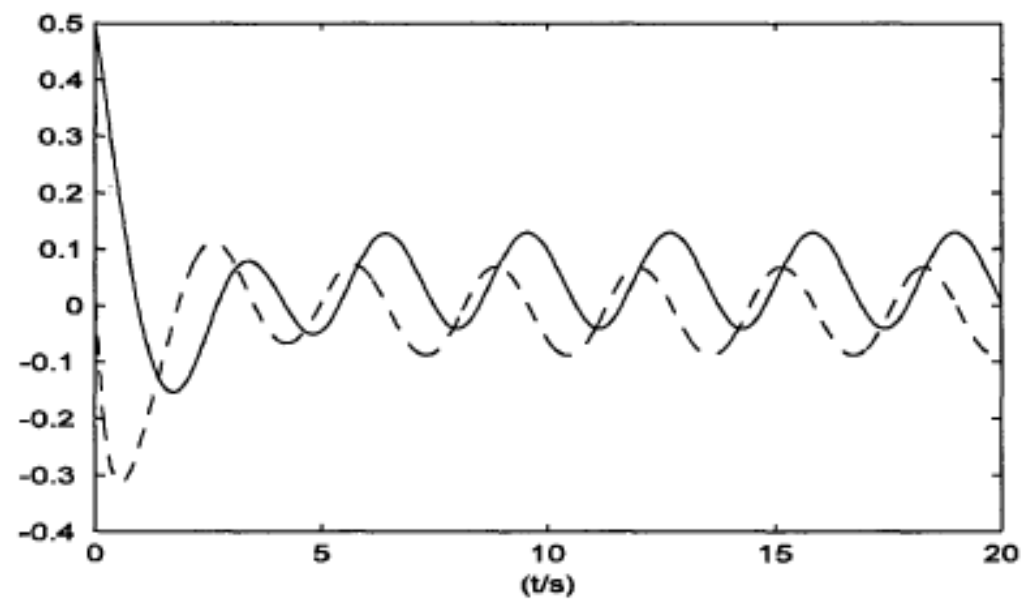

Fig.2 hypothetical result diagram

A Backstepping algorithm is proposed for a class of uncertain SISO system, and it is strict feedback of nonlinear. The Backstepping algorithm mainly includes two kinds of stable fuzzy control algorithms. Through the use of a RBF neural network approximator, the first algorithm is mainly on the assumption that the minimum approximation error meets the determined bounded conditions. Through the establishment of a special design scheme, it can effectively overcome the singularity problem of the controller, as a result, the performance of the closed-loop control system could be improved, and achieving the expansion of its scope. The second algorithm is based on the first algorithm, based on the fuzzy approximator, the online adjusting approximation of the unknown parameter vector bounded norm value, which will reduce the online computational burden. By choosing appropriate design parameters, the above mentioned two kinds of fuzzy algorithms can prove that all the closed-loop signals are uniformly bounded, and the tracking error converges to a small zero field.

As we all know, comparing to the strict matching condition system and feedback system, the system of pure feedback is more complex and general. Based on the application of the stability analysis method proposed by Lyapunov, a fuzzy control method for uncertain nonlinear stability class MIMO pure feedback systems has been proposed. This method has gradually relaxed the restriction of fhe literature for the model constraints. From Backstepping system, we learn that, at each step in the fuzzy system, it will be gradually approach to the unknown function of each subsystem of the approximation. Thus in the process of input design control, the robust control algorithm can effectively compensate the approximation error vector. The proposed control method must be in the closed-loop system for all signals use, only this way can the tracking error vector as well as the uniform bound of norm be in a small zero field convergence.

\section{Simulation Study}

Simulation research is an important work of research on fuzzy control of nonlinear system. This work is mainly divided into three steps. The first step is to improve the fuzzy algorithm diagram. Through the improvement of the system, the fuzzy algorithm will be stronger systematic. The second step is to make the data information into fuzzy algorithm diagram to realize operation control in practice to analyze the effect and performance of fuzzy control. The third step is to achieve further revision and improvement of the fuzzy algorithm diagram and it is based on the reflected effect and performance. In short, the simulation research is the application trial of actual fuzzy control algorithm for linear systems, and the actual application will be used to do the analysis of effect and performance, 
and there will be further trimming and adjustment according to the performance, so that the use effect of the algorithm is higher and the control performance is stronger. The analysis results show that the nonlinear system of fuzzy control algorithm has the following two remarkable characteristics in practice, the first one is it gets rid of the computation complexity, becoming much simple. The second one is that the algorithm achieves excellent control performance, and it is of great help to enhance control effect.

\section{Conclusion}

The computer control system is an important aspect of computer technology to enhance the research. From the research and practice of the control system, the effectiveness of control system of the accurately controlled object is easy, while mathematical modeling system will be difficult when encountered complex controlled object, and there will lots of problems in the practical controls. In order to solve this problem, it is significant to do positive research on fuzzy control, and it is also significant to deal with complex problems with fuzzy algorithm, finally it can make the problems simplified.

In summary, in the field of control theory, the control of nonlinear systems has been a very challenging issue. Through the study of two kinds of nonlinear structural systems, two kinds of effective fuzzy control algorithms were put forward in this paper. Because the nonlinear control input system exists in unknown nonlinear functions and some couplings between each subsystem, thus it is hard and complex to control the nonlinear systems, but also extremely difficult. By constructing some special structure and Lyapunov function, the mean value theorem, Backstepping design technology, fuzzy system application and the introduction of Nussbaum function, the above problems have been solved step by step. Based on the proposed methods, all of the signals in the closed-loop control system has been guaranteed in uniformly bounded situation. It can be seen that the feasibility of the design method is verified by the simulation experiment.

\section{References}

[1] Guo Feng. Research on predictive control algorithm of cement rotary kiln based on fuzzy ARX model [D]. Yanshan University, 2012

[2] Han Jingyuan. Study on the monitoring and hierarchical intelligent adaptive control algorithm for nonlinear cricket system [D]. Jilin University, 2014

[3] Wang Xudong. Research on fuzzy control algorithm based on inverted pendulum system [D]. Xi'an Electronic and Science University, 2012

[4] Li Xu. Direct adaptive fuzzy control of uncertain nonlinear systems [D]. Northeast Petroleum University, 2013

[5] Wang Bin, Qiu Zhicheng, Zhang Xianmin, Han Jianda. Adaptive Fuzzy Vibration Control of Flexible Arm Actuated by Pneumatic Drive [J]. Journal of Mechanical Engineering, 2013,11:50-60.

[6] Liu Wenlong. Multivariable Nonlinear Predictive Control Based on T-S Fuzzy Model [J]. Journal of Electronic Measurement and Instrument, 2013,10:998-1003.

[7] Dong Haiying, Wei Zhanhong, Zhao Xianggui, Li Xiaoqing, Cao Cao. Variable Universe Fuzzy Control [J]. Control Engineering Based on Multi Population Genetic Algorithm, Variable Universe Fuzzy Control Engineering, 2014,02:182-188.

[8] Zheng Lan, Zhou Weidong, Liao Chengyi, Cheng Hua. Adaptive Fuzzy Backstepping Predictive Control of Nonlinear Systems with Uncertain Nonlinear Systems [J]. Journal of Harbin Institute of Technology, 2014,11:107-111. 